\title{
Evaluación nutricional del paciente pediátrico hospitalizado
}

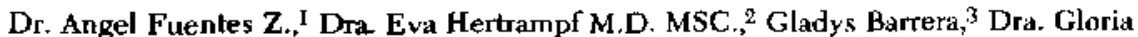
Heresi M.D. MSC., ${ }^{4}$ Dr. Sergio Jarpa ${ }^{5}$ Dr. Ricardo Uauy D. M.D.Ph.D. ${ }^{6}$

\begin{abstract}
A nutritional survey was conducted in order to establish the prevalence of malnutrion in patients from the medical and surgical wards. Data on anthropametric indicators of nutritional stahis were obtained on admission and at the tine of the sturly. They included weight $(W)$, height $(H)$, arnı circumterence $(A C)$ and triceps sknfold (TS). These values were expresed as of the reference standard lor age (A) (NCHS-USA). The study included 215 patients whose ages ranged from 2 months to 16 years, 58 were surgical and 157 medical patients; 113 were males and 102 females. On admission $25 \%$ were of nornal W/A but at the time of the study only $19 \%$ were in this range. Sixty seven \% of medical and $66 \%$ of surgical patients had a W/A less than $90 \%$ and $29 \%$ had Jow $\mathrm{H} / \mathrm{A}$ in both groups. The W/H was diminished in $42 \%$ of medical and in $15 \%$ of surgical patients whith a female predoninance of low W/H A.C. was below nomal in $65 \%$ of medical and in $43 \%$ of surgical patient; seventy three $\%$ of surgical patients presented T.S. below $90 \%$ of the stanchard. We arbilyzed the weight change of children under two sears and related it to the lenght of hospitalization finding that $72 \%$ of potients were losing weight and the rest were gaining at rates below the expected for their ages.

Based on this results we can conclude that there is a high prevalence of marasmic energy protuin inalnutrition. The length of hospitalization relates to a deteriocation of nutritional status. Patients present predominantly signs of depletion of the fat and muscle compartments.
\end{abstract}

\section{ABREVLATURAS USADAS}

AGB Area Grasa Braquial.

AMB Area Magra o Muscular Braquial.

CONIN Corporación para la Nutrición Infantil.

D.E.P. Desnutrición Energético Proteico.

INTA Instituto de Nutrición y Tecnología de los Alimentos.

PB Perimetro Braquial.

PCT Pliegue Cutáneo Tricipital.

P/E Peso Según Edad.

P/T Peso Según Talla.

T/E Talla Según Edad.

La evaluación nutricional del niño controlado ambulatoriamente por el Servicio Nacional de Salud (S.N.S.) en Chile revela tasas de Desnutrición

${ }^{1}$ Post Becado Pediatría General, Hospital Luis Calvo Mackenna. Becado Nutrición, Clínica Instituto de Nutrición y Tecnología de los Alimentos. Universidad de Chile.

2 Magister en Nutrición Humanze, Instituto de Nutrición y Teenologia de los Alimentos, U. de Chile.

${ }^{3}$ Enfermera del Centro de Investigación, Clínica Instituto de Nutrición y Tecrología de los Alimentos, Universidad de Chile.

4 Magister en Nutrición Humanł. Institutu de Nutrición y Tecnología de los Alimentos, U. de Chile.

5 Médico Jete Unidad de Nutrición, Hospital Luis Calvo Markenna. Santiago, Chile.

6 Jefe de la Lindad de Nutrición Clínica y del Centro de Investígación Clínica, Instihato de Nutricion y Tecuoblogia de los Alimentos, Universidad de Chile.
Energética Proteica (DEP) infantiles del 13\% en 1978 y $12 \%$ en $1979 .^{1}$ Siendo este tipo de desnutrición en su gran mayoria de tipo primario y en menor porcentaje secundaria a cuadros genéticos, metabólicos, endocrinológicos, gastroenterológicos y a enfermedades intercurrentes del tipo bacteriano o parasitario. De los desnutridos tratados por la Corporación para la Nutrición Infantil $(\mathrm{CONIN})^{2}$ un $4 \%$ corresponden a desnutridos secundarios. No se dispone de datos nacionales que revelen la incidencia de desnutrición primaria o secundaria en los pacientes pediátricos hospitaIizados.

En trabajos anteriores hemos aralizado los factores condicionantes de la destutrición intrahospitalaria y las técnicas para determinar la presencia de D.E.P. La evaluación del estado nutricional permite identificar el grupo objetivo en tel cual es imperioso prestar apoyo nutricional para disminuir estada hospitalaria, riesgo de infecciones y mortalidad asociada a desnutrición. Entre las mediciones utilizadas para la determiración del es. tado nutricional ${ }^{3}$ se incluye: historia nutricional (pérdida de peso e ingesta diaria), antropometria (talla, peso, perimetros y pliegues cutíneos), pro-

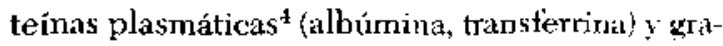
do de inmunocompetencia ${ }^{5}$ (pruebers sitúncas a antígenos PPD. Candida Albicians!.

Los objetivos del presente trabajo fueron: re:ahizar un diagnóstico de la situación mutricional del paciente pediatrico hospitalario. Evaluar la ne- 
cesidad de apoyo nutricional en este tipo de pacientes.

\section{PACIENTES Y METODOS}

En esta evaluación utilizamos las mediciones antropométricas disponibles de cada paciente en la iicha clínica al momento de ingreso, efectuánclose otras mediciones al momento del estudio gue se detallarán a continuación. Se encuestó a toda la población hospitalizadia en los servicios de Medicina y Cirugía del Hospital L. Calvo Mackenna el 8 de octubre de 1978. Se excluyeron a los pacientes prematuros y recién nacidos. De los 238 macientes hospitalizados se evaluaron 215 (91\%), lios 23 restantes correspondieron a 12 menores de 2 meses, 6 con edema de origen cardiovascular 0 venal y 5 por tener datos insuficientes. De la ficha clinica individual se tomaron los siguientes datos: edad, sexo, fecha y peso de nacimiento, servicio de hospitalización, diagnóstico principal y uno secundario. Previo a la encuesta se efectuó una calibración de las balanzas y antropómetros dis[onibles en las salas del hospital, detectándose un $30 \%$ de balanzas desequilibradas. En las mediciones de peso para este estudio se utilizaron aquellas balanzas que no tuvieran diferencias con los patrones de $500,1.000$ y $2.000 \mathrm{~g}$. Los antropónetros existentes no mostraron diferencias con los isados por el equipo encuestador (Infantometer 2867-1334 Graham Field). Cada paciente inmovilizado fue pesado desnudo y medido, en decúbito pasivo los menores de 24 meses y de pie los mayores, ${ }^{6}$ hasta el $0,1 \mathrm{~kg}$ y $0,1 \mathrm{~cm}$ más cercanos, respectivamente.

Para medir Perímetro Braquial (PB) y pliegues cutíneos se seleccionó a un grupo de 5 personas que efectuaron las mediciones con un coeficiente de variacion menor de $10 \%$ entre cada medición. El PB se midió con una cinta metálica y los pliegues con un calibrador de pliegues cutineos tipo Lange.

De los datos registrados se utilizaron los índices peso/edad, talla/edad, peso/talla; referidos a la nuediana de los estándares del NCHS - DHEW U.S.A., 1977. ${ }^{7}$

Las mediciones del PB y Pliegue Cutíneo Tricipital (PCT) fueron expresadas como porcentaje de la mediana, según edad $y$ sexo. ${ }^{7}$ De estas dos mediciones se derivó Area Magra Braquial (AMB) y Area Grasa Braquial (AGB), según las fómulas incluidas más adelante. AMB y AGB también fueron expresadas como porcentaje de la mediana de las tablas en referencia. ${ }^{8}$

$$
\begin{aligned}
\text { Area Magra Braquial }= & 0.07957(\mathrm{~PB}(\mathrm{~cm})-\pi \\
& \text { PCT }(\mathrm{cm}))^{2} \\
\text { Area Grasa Braquial }= & 0.5 \text { PCT } \times \text { PB }-0.78539 \\
& \text { PCT. } \\
\text { Donde PB }= & \text { Perímetro Braquial }(\mathrm{cm}) \\
\text { PCT }= & \text { Pliegue Cutaneo tricipi- } \\
& \text { tal }(\mathrm{cm}) .
\end{aligned}
$$

Los criterios revisados de Waterlow fueron utilizados para definir severidad del déficit de Peso para Talla, Talla para Edad y Peso para Edad. En base a estos criterios, todos los indicadores evaluados se dividieron en los siguientes rangos: Normal, 90 a $110 \%$ del Standard utilizado; Elevado, más de $111 \%$; Depletado Leve, 81 a $90 \%$; Depletado Moderado, 71 a 80\%; Depletado Severo, menos de $70 \%$ del Standard utilizado en la medición. Los déficit de Peso para Talla revelan una desmutrición aguda o de corta evolución, mientras que el déficit de Talla para Edad menor a $80 \%$ evalúa una desnutrición del tipo Crónico.

Albúmina plasmática fue determinada en 101 pacientes con el método de Rojkin y expresada como porcentaje de los estándares publicados. ${ }^{10} \mathrm{El}$ Microhematocrito se realizó mediante la técnica de Drabklin y como standard se usó en los niveles mediano por edad publicados por P. Dallman." La inmunidad celular se evaluó mediante pruebas cutáneas efectuadas en 142 pacientes $(60 \%)$ que mostraron cicatriz a BCG en el hombro izquiendo. A cada uno de estos pacientes se inoculó $0.1 \mathrm{ml} 2$ U PPD (Preparados por el Instituto Bacteriologico de Chile) en antebrazo izquierdo y $0.1 \mathrm{ml}$ Antígeno de Candida (Laboratorio Hollister-Steir Yeardor Philadelphia. USA) en dilución 1:100 en antebrazo derecho. $\mathrm{L}_{\mathrm{a}}$ respuesta al antígeno inoculado se consideró positiva si la zona indurada encontrada a las $\mathbf{4 8}$ horas era mayor de $5 \mathrm{~mm}$ leída en sentido transversal.

Los datos fueron transcritos de la hoja de encuesta a una hoja de computación para ser posteriomente perforadas en tarjetas y almacenadas en una cinta para comparación con posteriores eshudios. Las variables brutas y transgeneradas fueron analizadas en un computador IBM 370 mediante programas estadísticos de IBM (5796 - PGQ for APLSV and APL/CMS y 5796- PHW for VSAPL. ${ }^{12}$

La distribución de las edades de los 215 pacientes fue la siguiente: 98 (45\%) menores de 1 año, 20 
19\%) I a 2 años, 42 (19.5\%) 2 a 6 años, 32 (15\%) 6 a 10 años, 23 ( $11.5 \%$ ) 10 a 16 años; 15.5 (72\%) hospitalizados en el Servicio de Medicina y 60 (28\%) en el Servicio de Cirugía, 113 fueron hombres y 102 mujeres. Del grupo encuestado, 39 (18\%) estalban hospitalizados más de 7 dias, $40(19 \%)$ más de 14 dias y $44(20.5 \%)$ más de 4 semanas.

Los 196 pacientes con peso dé nacimiento mavor ipue el percentil $5^{7}$ fueron clasificados segun Peso/Edad en los gnupos descritos. En 161 pacientes perados al ingreso se utilizó lia diferencia con el peso durante el período de hospitalizacion.

\section{RESULTADOS}

El diagnóstico principal consignado en la ficha clínica permitió agrupar a los pacientes en las categorías observadas en la Tabla l. Existe un predominio de las infecciones respiratorias y enfermedades infecto-contagiosas (56.6\%) sobre las otras categorías médicas, cardiopatías $(9.6 \%)$, enfermeditcles oncológicas $(8.9 \%)$, digestivas $(8.3 \%)$, desnutridos (4.5\%). En los pacientes quirírgicos. zur $33 \%$ son de emergencia, traumatología (24\%). cirugía ortopédical $(20 \%)$ y cintgía reparadora (12\%).

La Figura 1 muestra las características del grupo total para los indicadores antropométricos y bioquímicos evaluados. El porcentaje promedis \pm D.E. de $\mathrm{P} / \mathrm{E}$ al ingreso fue de $82 \pm 18 \%$, disminuyendo significativamente al momento del estudio, que en promedio correspondióa 13 días, a 78 $\pm 16 \%(\mathrm{p}<0.05)$. Talla/Edad en promedic) fue 93 $\pm 12 \%$, acercándose más a lo nonnal y. P/T se balla levemente disminuido $39 \pm 16 \%$.

Tabla I

Distrilłución de pacientes según diagnósticos H.I.C.M. 1978

\begin{tabular}{|c|c|c|}
\hline Médicoss & $\mathrm{N}$ & $\%$ \\
\hline - Respiratorios & 66 & 42 \\
\hline - Infecto-contagiosas & 23 & 14,6 \\
\hline - Cardiovaseulires & 15 & 9,6 \\
\hline - Oncológicicos & 14 & 8,9 \\
\hline - Digestivos & 13 & 8,3 \\
\hline - Desmutridos & 7 & 4.5 \\
\hline - Hematalógicos & 4 & 2,5 \\
\hline - Nefralógicos & 4 & 2,5 \\
\hline - Venrológicos & 4 & 2,5 \\
\hline - Cenéticoss-metabólicos & 3 & 1,9 \\
\hline - Mesenquimopatias & 3 & 1,5 \\
\hline - Intoxicación salicílica & 1 & 0,6 \\
\hline TOTAL. & 157 & 100 \\
\hline \multicolumn{3}{|l|}{ Quirirgicos } \\
\hline - Aguoles & 17 & 33 \\
\hline - Traumatológicos & 12 & 24 \\
\hline _- Ortopédicos & 10 & 20 \\
\hline - Plísticos & 6 & 12 \\
\hline — Urolögicos & 3 & 6 \\
\hline - Digestivos & 2 & 3 \\
\hline -- Toraicicon & 1 & 2 \\
\hline TO'TAL & 51 & 100 \\
\hline Sin diagnóstico & 3 & \\
\hline
\end{tabular}

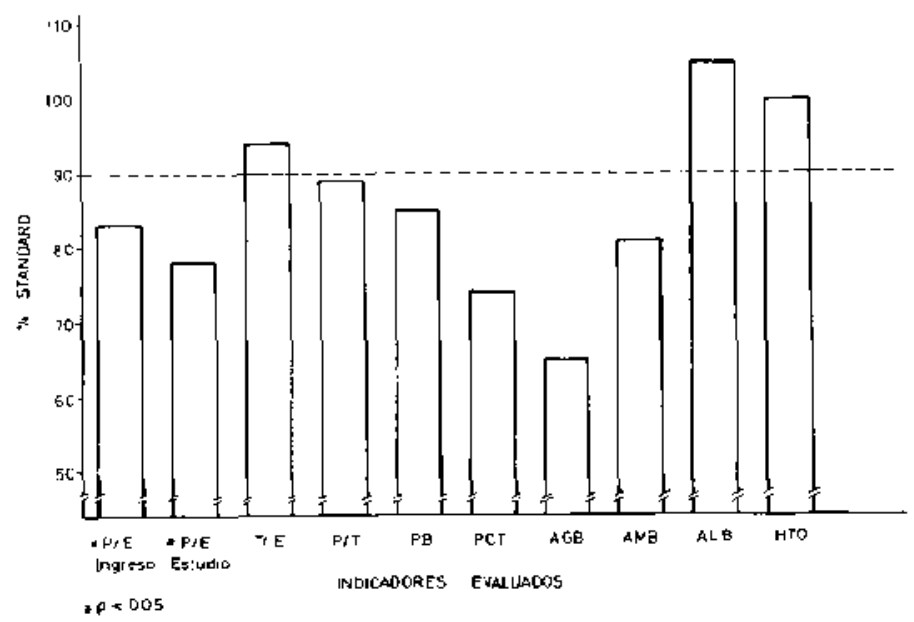

Figura I. Indicadores antropernétricos y bioxpusmicoss e'valuados. La aithra de cucda barm representa el promedir sel indkadsr evaluado con respecto al poremtaje del standarcl.

$\mathrm{P} / \mathbf{E}=$ Peso según Edad, T/E = Tialla según Ediarl. PirT $=$ Peso seyún Talla, $\mathrm{PB}=$ Perímetro Brarjuial, $\mathrm{PCT}=$ Pliegue Cutineo
Tricipital, AC:B = Area Grasa Bracpuial, AMB = Area Mapra Brạuial, ALB = Albimisal Pfismática, HTO = Hematocrito. La lírtea punteadit en el 9oo seibala el Ifrrite interior dt:l nugo normal (90-1 10\%). 
Los indicadores antropométricos braquiales, P.B. fie en promedio $85 \pm 15 \%$ y PCT $74 \pm 25 \%$ revelaron un compromiso importante en los compartimentos magro $y$ graso periféricos, los cuales se pueden cuantificar más precisamente al caluular las áreas magras $82 \pm 26 \%$ y grasa del brazo 65 $\pm 31 \%$, mostrando mayor compromiso de las reservas grasas que de las musculares. El indicador del compartimento magro visceral, la albumina plasmatica $104 \pm 14 \%$; y hematocrito $98 \pm 12 \%$ estaban nomales, en promedio, caracterizando al grupo en general como pacientes con denutrición predominante del tipo marásmica.

\section{- pesct eoad}

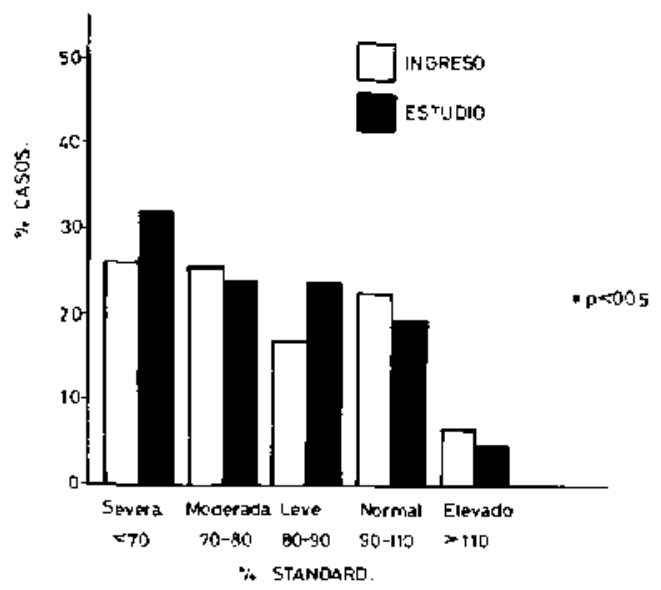

Figara 2. Distribución sle Peso según Edact en el momento de Ingreso y Estudio en pacientes Médicos y Qurúrgicos. J al alturat de las barras represenca el porcentaje de casos $\left(^{*}=0<0.05\right.$ ). representa la diferencia de los pronedios en los dos momentos.
La Figura 2 muestra la distribución de pesof edad al momento del ingreso y al estudio para 161 pacientes médicos y quirúrgicos. Los casos en el grupo de desnutridos leves aumentan de $19 \%$ a $23 \%$, de 26 a $32 \%$ en el grupo de desnutridos severos, disminuyen de 23 a $19 \%$ los considerados nonnales y de $6.8 \%$ baja a $4.8 \%$ el grupo con $\mathrm{P} / \mathrm{E}$ elevado. El grupo con desnutrición moderada se mantiene casi constante, en un $25 \%$, esto se explica por igual número de pacientes que pasan del grupo desnutrido leve al moderado y de moderado a severo. De 62 pacientes que ingresan normales, P/E mayor de $90 \%, 21$ se desnutrieron al momento del estudio y de los 71 desnutridos al ingreso, $\mathrm{P} / \mathrm{E}$ menor que $50 \%$, sólo 4 se habian recuperado al momento del estudio $(\mathrm{p}<0.001)$. Según estos criterios, sólo el $35 \%$ de los pacientes tenian un estado nutricional normal con $\mathrm{P} / \mathrm{E}$ mayor al $90 \%$ de standard y existía un $33 \%$ de los pacientes con $\mathrm{P} / \mathrm{E}$ bajo $70 \%$ del standard, en contraste con el $4.5 \%$ consignados como desnutridos en la ficba clinica.

La distribución de los pacientes médicos y quirúrgicos según $P / T$ y $T / E$ se aprecia en la Figura 3.

Là distribución según talla para edad no muestra diferencias significativas en ambos servicios, contrastando con lo encontrado en la distribución de $\mathrm{P} / \mathrm{T} ; 60 \%$ de los pacientes médicos tienen $\mathrm{P} / \mathrm{T}<$ $90 \%$, contra un $30 \%$ de los quirúrgicos ( $\mathrm{p}<0.05$ ). Estos resultados sugieren predominio de desnutrición aguda en los pacientes médicos del hospital. Según los criterios de Waterlow (Tabla 2),
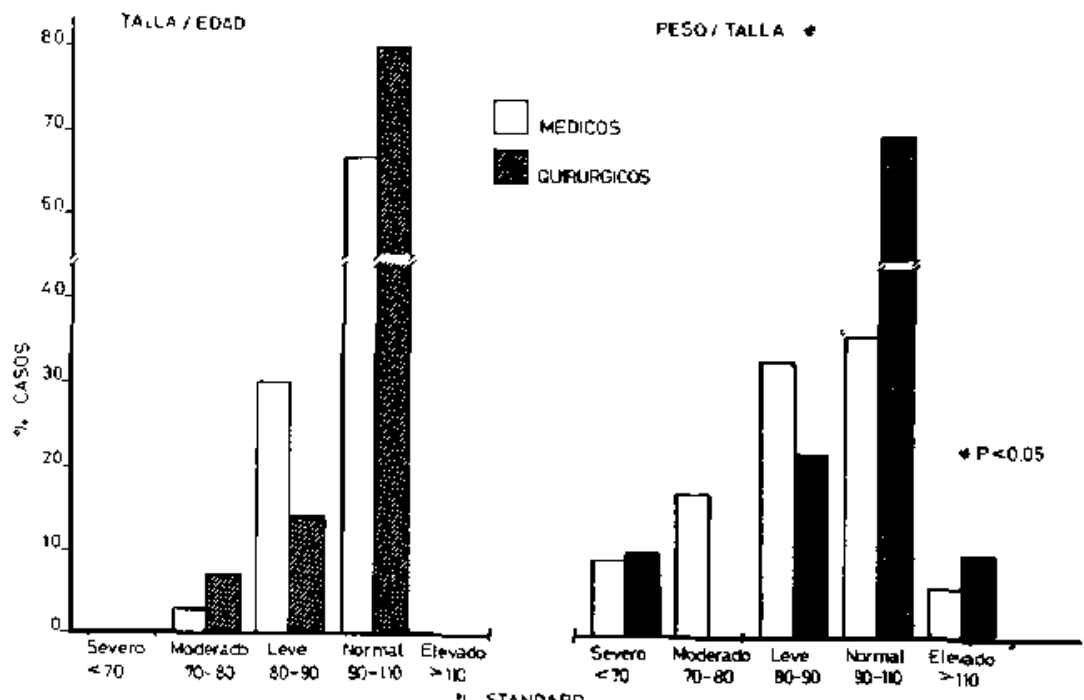

Figura 3. Distribución de pacientes médicus y quirúrgicos seguin Talla/Edad y Peso/Talla: la altura de las barras representa el porcentaje de casos y el eje horizontal los grados de desnutrición separado en rangos. El asterisco representa diferencla significativa entre pacien. tes médicos y quirúrgicus. 
$51.2 \%$ de los pacientes tuvieron evidencias de desnutrición aguda expresada por la relación $P / T$ menor de $90 \%$ y de éstos, 15 (10\%) tuvieron desnutrición aguda de 3er. grado. Casi el $60 \%$ de los pacientes tenían desnutrición crónica evidenciada por la relación Talla/Edad menor al $95 \%$ del standard.

La distribución de los indicadores antropométricos braquiales se presenta en la Figura 4.

Al comparar los pacientes médicos con los quirirgicos, los primeros muestran niveles de $\mathrm{PB}$, AMB y AGB significativamente menores que los quirúrgicos ( $p<0.01$ ), no existen diferencias al compararlos según PCT.
Tabla 2

Incidencia de desnutrición según criterio de Waterlow. Hospital Luis Calvo Mackenna, 1978

\begin{tabular}{crrrrrr}
\hline \multicolumn{5}{c}{ \% de pacientes $(164)$} & \\
\hline Grado & & Aguda & Grado & & Crónica \\
& $\mathrm{P}^{\prime} \mathrm{T}^{*}$ & & & $\mathrm{~T} / \mathrm{E}^{*}$ & \\
0 & $>90 \%$ & 49.0 & 0 & $>95 \%$ & 40.0 \\
1 & $\mathbf{8 1 - 9 0 \%}$ & 29.0 & 1 & $90-95 \%$ & 31.0 \\
2 & $71-80 \%$ & 13.0 & 2 & $85-89 \%$ & 18.0 \\
3 & $<70 \%$ & 9.0 & 3 & $<85 \%$ & 11.0 \\
$1-3$ & & 51.0 & $1-3$ & & 60.0
\end{tabular}

*Porcentaje del standard NCHS-LSA. 1978.

Figura 4
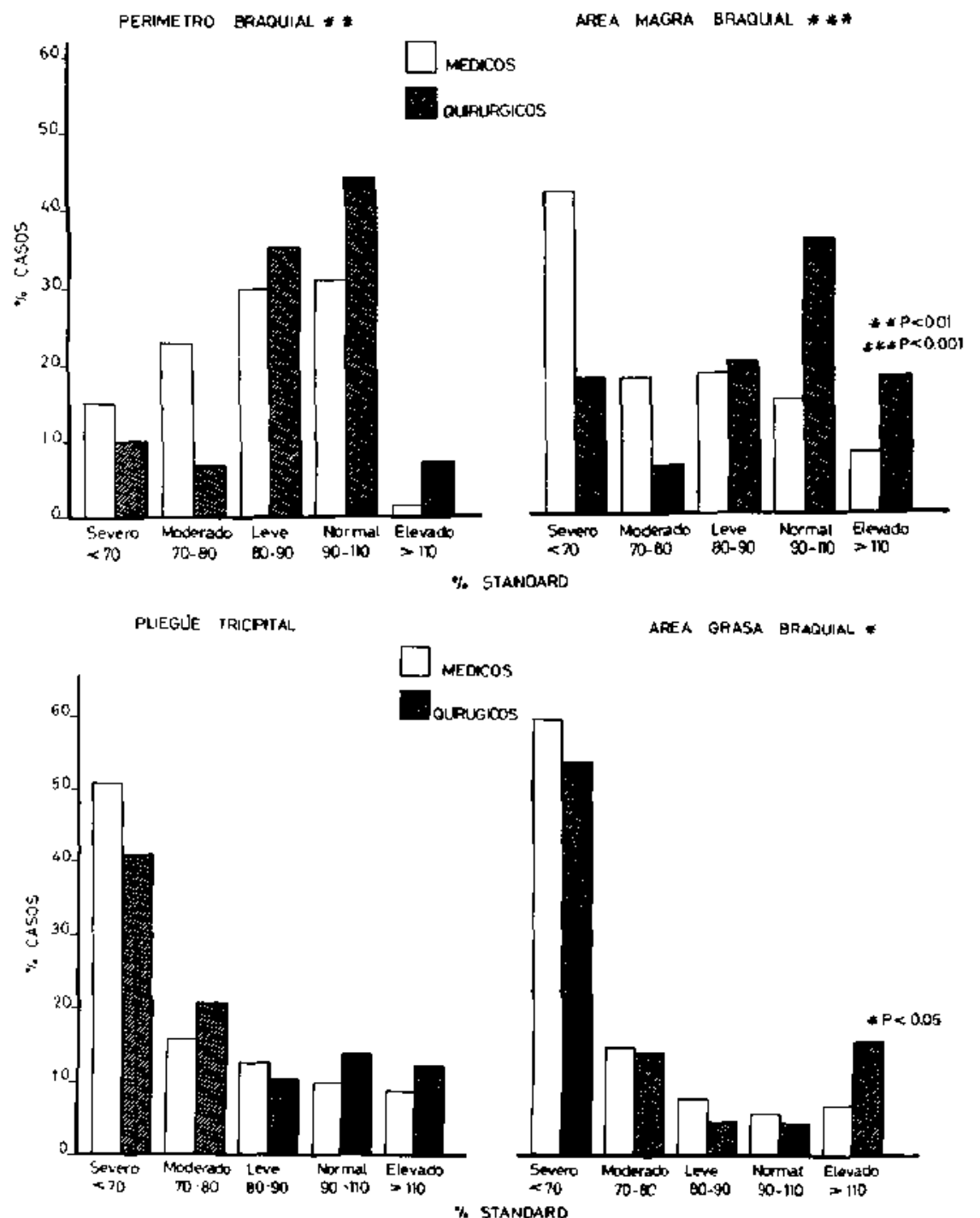

Figure 4. Distribución rle Indicadores Braquiales en pacientes médicos y (uirúrgicos. Según Perinetro Braquial (a), Area Ma. (juial (d), Id altura de cada barra representa el porcentaje de los cuasos. Los asteriscos representan las diferencias de promedios según t de Student. 
El $4 \mathrm{~L} \%$ de los pacientes médicos y el $18 \%$ de los pacientes quirírgicos se hallan severamente depletados en área inagra braguial y existe un $50 \%$ de pacientes médicos y quirírgicos con depleción severa de la grasa bratuial, definiendo depleción severa por valores menores alue $70 \%$ del standard.

En la Tabla 3 se describe la prevalencia de hematocritos bajos comparíndola con el ECEN (Encuesta Continuada del Estado Nutricional, 1974). El gnipo de lactantes presenta una proporción de hematocritos bajos semejante a la población general, a diferencia de los mayores de 2 años, que es varias veces mavor que lo considerado nonnal patria nuestro medio.t.

Tabla 3

Prevalencia de hematocritos bajos segín edad. Hospital I mis Calvo Mackenna. 1978

\begin{tabular}{cccc}
$\begin{array}{c}\text { Edad } \\
\text { (años) }\end{array}$ & $\begin{array}{c}\text { Limite normal } \\
\text { HTCO) }\end{array}$ & $\begin{array}{c}\% \text { HTCO. bajos } \\
\text { HCLM }\end{array}$ & ECEN* \\
\hline $0.5 \cdot 2$ & 33.0 & 21.4 & 28.1 \\
$2-4$ & 33.0 & 27.6 & 16.8 \\
$4-8$ & 34.5 & 19.4 & 6.7 \\
$8 \cdot 11$ & 36.0 & 40.0 & 6.7 \\
$>11$ & 37.0 & 16.6 & 4.6 \\
\hline
\end{tabular}

*Encuesta Continmada de] Estado Nutriciona] - 1974.

pRUEEAS CUTANEAS

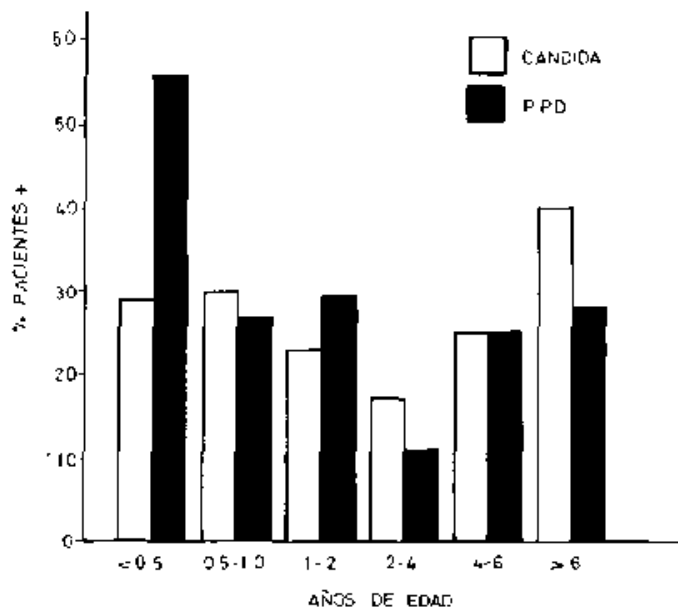

Figura 5. Distribución de (wacientes BCX; +) segini edad en

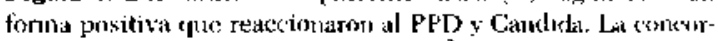
datucia ile ambats groeljas fite de $65 \% 0\left(x^{2}=6.72\right)$.

Lat Figura 5 muestra la distribución etaria de 142 pacientes que reaccionaron a las pruebus cutáneass al PPD y Candida. Eu general, sólo el 34\% de los pacientes inoculados reaccionaron al PPD $y$ el $32 \%$ al antígeno de Candida. Existió una concordancia significativa en la respuesta a estas dos pruebas $(p<0.001)$. En la Tabla 4 se observa la relación existente entre pruebas cutáneas y depleción del área magra braquial, tomando como linite menos que el $80 \%$ del standard; 50 de 81 (51\%) pacientes con ANB baja no respondieron a Ios antígenos inoculados, contra 36 de 103 pacientes $(34 \%)$ con AMB aceptable $(p<0.01)$.

Tabia 4

Relicion entre priebas cutaneas y áred metgra bracuial. Hospital Luis Calvo Mackenna. 1978

\begin{tabular}{|c|c|c|c|}
\hline \multicolumn{4}{|c|}{ Pruebas cutáleas* } \\
\hline Yo standard & $\begin{array}{l}\text { Lrat o muts } \\
\text { positiva }\end{array}$ & $\begin{array}{c}\text { Ambas } \\
\text { negativas }\end{array}$ & Total \\
\hline$<80$ & 31 & 50 & 81 \\
\hline \multirow[t]{4}{*}{$=80$} & 67 & 36 & 103 \\
\hline & 98 & 86 & 184 \\
\hline & & $x^{2} 14.9$ & \\
\hline & & $p<0.01$ & \\
\hline
\end{tabular}

Tabla 5

Distrilunción de patcientes médicos y cuainirgicos segün albuisrina plasmáticut. Hospital duis Calvo Mackenna. 1978

$\begin{array}{cc}\text { Médicos* } & \text { Quinirgicos* } \\ (126) & 154 !\end{array}$

\begin{tabular}{|c|c|c|}
\hline $\begin{array}{c}\text { Albúmina } \\
\text { \% del standirdi** }\end{array}$ & $\%$ & $\%$ \\
\hline$<70$ & 0.8 & 0.0 \\
\hline $70-80$ & 1,6 & 5.5 \\
\hline $80-90$ & 4.0 & 22,2 \\
\hline $9(0)-110$ & 60.3 & 48.1 \\
\hline $110 \cdot 120$ & 190 & 20.3 \\
\hline \multirow[t]{2}{*}{$>120$} & 14.3 & 3.7 \\
\hline & $1(k) .0$ & 10030 \\
\hline Pronuedios \pm E.S. & $106 \pm 1.2$ & $99 \pm 1.8$ \\
\hline
\end{tabular}

En la Tabla 5 se aprecia la distribución de la albúmirsa en 180 pacientes médicos y quirirgicos. Existe diferencia significativa entre la albúmisıa 
sérica promedio de los pacientes médicos $106 \pm$ $13.5 \%$ y quirúrgicos $99 \pm 13.2 \%$ ( $<<0.01)$, destacándose que un $27.7 \%$ de los pacientes tienen albúminas menores al $90 \%$ del standard. En la Tabla 6 se presenta la relación encontrada entre albifrnina plasmática y duración de la estada en 101 pacientes médicos. El 3\% de los pacientes hospitulizados por menos de un mes tienen albúminas bajas, mientras que en los hospitalizados por más de 30 días ello ocurre en un $26.7 \%$. Esta diferenciat es significativa al 0.001.

Tabla 6

Rèación entre albúmina plasmática y rluración estada en pacientes médicos. Hospital I uis Calvo Mackenna. 1978

\begin{tabular}{|c|c|c|c|}
\hline \multirow{3}{*}{$\begin{array}{l}\text { Estakla } \\
\text { (días) }\end{array}$} & \multirow{3}{*}{$\mathbf{N}$} & \multicolumn{2}{|c|}{ Albuimina } \\
\hline & & $B$ iaja* & Nommal \\
\hline & & \multicolumn{2}{|c|}{ \% Pacientes } \\
\hline$<30$ & 86 & $3.3^{* *}$ & 96.7 \\
\hline$>30$ & 15 & $26.7 * *$ & 73.3 \\
\hline
\end{tabular}

${ }^{*}$ Baja < $90 \%$ del standard (Hitzig, 1963).

* $\mathrm{p}<0.001$ ditcrencia en albúmina según estadas.

\section{DISCUSION}

Como el objetivo de la investigación fue detectar la prevalencia de desnutrición en el ambiente hospitalario infantil, utilizamos los criterios de Waterlow, debido a que diferencian adecuadamente niños desnutridos o depletados en fonma aguda de los depletados en forma crónica. Según estos criterios, encontramos un $51 \%$ de pacientes mayores de 2 meses depletados agudos y un $60 \%$ de pacientes depletados en forma crónicu, estos últimos ingresaron ya desnutridos al hospital, Los standards antropométricos utilizados correspondieron a las tablas del NCHS - USA 1977, recomendadas por la OMS ${ }^{14}$ cono referencia en estudios transversales, además representan valores de población con su potencial de crecimiento genético no modificado por factores ambientales.

En los resultados de la encuesta llama la atención un 4.5\% de desnutrición, según criterio clínico, al tabular los dos primeros diagnósticos de la ficha clínica, esto podría revelar un criterio de diagnóstico netamente curativo que es necesario modificar hacia lo preventivo con el fir de detectar precozmente a la población hospitalizada en grave riesgo nutricional. Sin embargo, la evaltación precisa de estado nutricional realizada en el presente estudio muestra que sólo un tercio de los pacientes tienen una situación normal y que uno de cada tres tienen un déficit importante de peso para la edad. La alta prevalencia de desnutrición en el uredio hospitalario es de esperar, dadas las múltiples interacciones entre nutrición y salud. No tan sólo la enfernnedad condiciona desnutrición, sino cue la incidencia y letalidad de diversas enfermedades es significativamente más alta en el desnutrido. Es de especial interés verificar la gran prevalencia de enfennedades infecto-contagiosas en la población estudiada, ya que ello apoya los inúltiples estudios de interacción entre nutrición e infección. ${ }^{25} \mathrm{Al}$ respecto, el estudio subre causas de mortalidad de la infuncia en América Latina evidenció que menos del $5 \%$ de las muertes terian el diagnóstico de desnutrición, sin embargo, más de un $50 \%$ se debían a enfenmediades infeciciosas en las cuales la desmutrición cletemrina at tas tasas de letalidad. ${ }^{2 h}$ Es así cono el sarampión tiene una letalidad 300 veces más alta en el desnutrido de tercer grado. Si bien el factor nutricional no es el agente etiológico, es el mayor determinante de la muerte.

El tipo de desnutrición encontrada fue principalmente del tipo marasmo agudo, algunos pacientes podrian ser catalogados como marasmo-kwashiorkor por tener compromiso pondoestatural y niveles de albúmina levemente comprometidos, no detectamos pacientes quirirgicos con kwashiorkor clínico franco. La velocidad de desnutrición (pérdida diaria de peso expresado como \% del standard) equivalente a $0.3 \%$ diario por paciente sugiere que, de mantenerse, se puede perder un $10 \%$ de peso al mes y que el hospital contribuiria a generar desmutridos con el consecuente aumento en el riesgo de enfermar especialmente por patología infecto-contagiosa. Este estudio no pretende mostrar la incidencia de desnutrición en el hospital, ya que se efectuó exclusivamente un corte transversal. Sin embargo, la distribución de pesoledad en el momento del estudio se desvió hacia niveles más bajos de adecuación con respecto al standard, esto equivale a aumentar en $6 \%$ la tasa de desnutrición severos y en $4 \%$ el porcentaje de desnutridos leves con respecto al ingreso.

El índice Peso/Talla detectó un $10 \%$ de pacientes médicos y quirúrgicos severamente depletados, por tener un déficit de $20 \%$ o más en el peso correspondiente a su talla real. Se destacó tum- 
bién la nonmalidad de los pacientes quirúrgicos frente al parámetro Talla/Edad, lo que revela la presencía de población enferma agudamente que no ha comprometido su crecimiento estatural, con una estatura comparable a lo normal según las tablas norteamericanas de referencia, validando su uso en este tipo de estudios. ${ }^{15}$ Los índices braquiales AGB y PCT determinaron en forna aproximada el compromiso del compartimento de las reservas energéticas del tipo graso y se establecen diferencias significativas entre pacientes médicos, más depletados y los quirúrgicos.

Los resultados de positividad al PPD y a la Candida son bastante más bajos que los esperados para las respectivas edades. En trabajos realizados por el INTA (datos no publicados) y por otros, ${ }^{16}$ alrededor de un 55\% de los lactantes eutroficos presentaron reacción cutánea positiva con $2 \mathrm{U}$ y 10 U de PPD, respectivamente. En trabajos internacionales, más de un $80 \%$ de los niños entre 6 meses y 5 años tienen reacción positiva al antígeno de Candida. ${ }^{7}$ La literatura presente concuerda con este estudio señalando que la desnutrición negativiza las pruebas cutáneas, esto también se observa con alguin tipo de infecciones. ${ }^{18,}{ }^{19}$ En este trabajo, un $\mathbf{4 8 \%}$ de los niños presentaron el diagnóstico de infecciones al momento del estudio, lo cual puede estar contribuyendo a la negativización de estas pruebas. Sin embargo, la relación encontrada entre el Area Magra Braquial y la anergía en la inmunidad celular apunta al factor nutricional como componente etiológico importante. El Area Magra Braquial se revela como indicador útil del nivel de depleción visceral, lo cual permitiría en forma rápida seleccionar aciuella población que necesita apoyo nutricional intensivo utilizando las ténicas disponibles. La asociación entre albúmina plasmática y estada mayor de 30 días plantea la necesidad de controlar el parámetro periódicamente a fin de detectar a los pacientes que han comprometido su sintesis proteica visceral en que posiblemente la desnutrición puede estar contribuyendo a prolongar la enfermedad.

El $27 \%$ de pacientes quirúrgicos con niveles de albúmina menores al $90 \%$ del standatd (3.2 - 3.4 $\mathrm{gr} / \mathrm{dl}$ ) reafirma en parte los hallazgos encontrados en pacientes adultos quirúrgicos chilenos ${ }^{21}$ y norteamericanos, ${ }^{22}$ lo que se explica por el mayor requerimiento proteico de estos pacientes derivados de la mayor síntesis proteica tisular que participa en la reparación y cicatrización de las heridas operatorias. En un estudio paralelo realizado en este hospital ${ }^{23}$ se analizó la adecuación de la ingesta y se demuestra que los pacientes que cubren menos del $90 \%$ de sus requerimientos energét$\cos$ y proteicos pierden peso en forma significativa al compararlos con los que ingieren más de 90\% de lo recomendado para individuos sanos.

Resumiento entonces, las dos variables en la producción de la desnutrición energético-proteica en el ambiente hospitalario son: la presenciaseveridad del stress y la presencia-severidad de la restricción proteico-energética. De tal modo que cuando las necesidades energéticas no son satisfechas por la dieta, el déficit calórico debe cubrirse utilizando una combinacion de los 3 compartimentos de reserva energética, músculo esqueletico, proteína visceral y tejido graso. La contribución relativa y actual de cada compartimento tisular detenmina el tipo y severidad de la desnutrición energético-proteica resultante. ${ }^{24}$ Esto se puede evitar mediante un programa de apoyo nutricional consistente en evaluación acabada del estado nutritivo al ingreso del paciente, dieta de acuerdo a los requerimientos energético-proteico individual concordante con el tipo de patología del paciente, monitoreo periódico del estado nutricional a fin de detectar desnutrición oportuna y precozmente y utilización adecuada de técnicas de apoyo nutricional enteral y parenteral de acuerdo a conocimientos modernos de nutrición clínica.

\section{CONCLUSIONES}

La prevalencia de la desnutrición energético-proteica en el ambiente hospitalario infantil supera el $50 \%$ de los pacientes y existe un $60 \%$ de pacientes con desnutrición crónica. A mayor tiempo de hospitalización aumenta el compromiso del estado nutricional. A la semana aumentan las tasas de desnutrición en $10 \%$ y al mes ya se aprecta compromiso del compartínento magro visceral. La desnutrición energético-proteica del paciente hospitalizado compromete principalmente el compartimento graso, magro-muscular y parcialmente el magro-visceral albúmina y pruebas cutáneas. Las consecuencias de la desnutrición intrahospitalaria en el estado inmunitario y síntesis proteíco-visceral permiten recomendar la necesidad de establecer medidas de apoyo nutricional para disminuir morbilidad asociada y acortar tiempo de recuperación. 


\section{AGRADECLMIENTOS}

Expresamos nuestros sinceros agradecimientos a todas las personas que hicieron posible la realjzación de este estudio:

-Al Dr. José Zacarías y su grupo de becados e internos.

- A la Sra. Irene Trufello, en la etapa de análisis de datos e inferencia estadística.

-A las secretarias, Srtas. Ana María Walsen y Genoveva Escobar.

-Al Sr. Gustavo Puente, por la excelente calidad de sus gráficos.

$\mathrm{Y}$ a todos quienes con su inestimable paciencia $y$ amistad contribuyeron en mayor o menor grado al éxito de esta labor.

\section{REFERENCLAS}

${ }^{1}$ Ministerio de Salud Pública, Chile, 1979.

2 Informes trinestrales de Conin al SNS, $197 \mathrm{Y}$.

${ }^{3}$ Elackbum, G. L.; Bistrian, B. R.; Maini, B. S.; Schlam, H. J., and Smith, M. F. Nutritional and metabolic assessment of the hospitalized patient. J. Parenteral Enteral Nutr., 1: 11.1977.

${ }^{4}$ Russell, J.; Merrith, Robert: Suskind, M. Nutritional Survey of hospitalized pediatrics patieuts. Amt. J. Clin. Nutr., 32; 1320-1325, 1979 .

$5_{\text {Latc, }}$ D. K, Dudrick, S. J., and Abdou, N. I. Inum Inocompetence of patients with F.C.M.: The effects of nutritional repletional. Aon Internal Med 79; 545, 1973.

6Jellife, $D$. $B$. The assessment of the nutritional status of the community. Geneva, WHO Monograph 53, 1966.

${ }^{7}$ NCHS Growth Curves for Children, Birth-18 years. DHEW Publication No (PHS) 78-1650 Series 1 l-N. 165.

${ }^{b}$ Frisancho, A. R. Triceps Skinfold and upper arm nulscle size norms for assessment of nutritional status. Am. J. Clin. Nutr, $27 ; 1052,1974$
"Waterioni, $J$. Some aspects of childhood malnutrition as at public health problem, Brit. Med. J. 4; 88, 1974.

L0 Scientific Tables (7."Edition 1970). Ed. K. Diem, C, Lewtner, Dicumentza Geigy.

"Dallmun, P. R.; Sumes, M. A. Percentile curves for themuglobin and red cell volume in infant and childhood $\mathrm{J}$, of Pediatrics 94 (1) 26-31, 1979.

${ }^{12}$ lnstalled User Pmgrant SH20-184 1-0. (1 ${ }^{\circ}$ Ed. L976) IBM.

${ }^{13}$ ECEN. Encuest. Continua del Estado Nutricional. Ministerio de Salud, 1974.

14 Measurement of nutritional impact pag. 4\%HO/FAE/79.1.

15 Monckebers, F.; Donoso, G.; Vatiente, S.; Arteaga, A.; Maccioni. A.i Merchak, $N$. Estudio del estado nutritivo y de lav condiciones de vida de la poblavión intantil en la provincia de Curico. Rev. Chil. Ped. 38: 491, 1967.

If Willshuw, M. E.; Suntiago, R.; Infinte, R., y Vargas, Marta Duración de la alergia induçida por BCC; Rev. Chil. de Pedlittria 87: 174, 1966.

17 Shumm, D. C.; Johnson, G.; Rusen, F. S., and Austen, K. F. Cellular reactivity to candida albicans antigens. New Engl. Joumal of Med. 275: 690, 1966.

IM Suskind, $R$. M., ed. Malnutrition and the immume response, New York, Haven Press, 1977.

${ }^{6}$ Chandro, $\boldsymbol{R}, K$, aud Newberne, P. M. Nutrition, imınınity and infection mechanisus of interactions. New York. Plentun Press, 1977

${ }^{20}$ WHO Scientific Group. Immumodeficiency Tech. Rep. Ser. 630, World Health Onganization, Genevit, 1978.

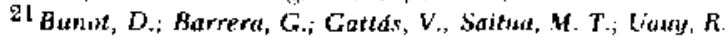
Estiado mutritivo de sujetos adultos hospitalizados. Un estudio transversal. Rev. Med. Chile 108: 700, 1980.

${ }^{22}$ Gristian, B. R.; Blackbum, G. L.; Hallotiell y col. Protein Status of general surgical patients. J.A.M A. 230: 855, 1974.

${ }^{23}$ Gattás, Y., Fuentes, A.; Jarpa, S., Clauy, R. Situdción alimentaria de pacientes pediátricos hos pitalizados (por publkar).

${ }^{24}$ Bristian, Brtce $R$. Interaction of nutrition atud intection in the hospital setting, Am. J. Clin. Nutr, 30: 1229-1232, 1977.

25 Scrimshaw, Nevin S. Effect of infection on nutrient requirements, An. J. Clin. Nutr, 30; 1536-1544, 1977.

26 Puffer, $R, R$, and Serrano, C. V. (1973). Pattem of inortality in childhood. PAHO Sci. Pub., N. ${ }^{\circ}$ 262. XLX Wash., D.C.

27 Uatuy, R.; Gasitéa, R. Evaluación del estado nutricional del entertuo hospitalizado. Rev. Méd. Chule 108: 542, 19840. 